\title{
CLINICAL AND MICROBIOLOGICAL PROFILE OF CANDIDA ISOLATES FROM ORAL CANDIDIASIS IN PATIENTS UNDERGOING RADIOTHERAPY FOR HEAD AND NECK MALIGNANCY
}

\author{
MRIDULA MADIYAL ${ }^{1}$, KRISHNA SHARAN ${ }^{2}$, INDIRA BAIRY ${ }^{3 *}$, PRAKASH PERALAM YEGNESWARAN ${ }^{1}$, \\ VIDYASAGAR MS ${ }^{2}$
}

${ }^{1}$ Department of Microbiology, Kasturba Medical College, Manipal University, Manipal, Karnataka, India. ${ }^{2}$ Department of Radiotherapy and Oncology, Kasturba Medical College, Manipal University, Manipal, Karnataka, India. ${ }^{3}$ Department of Microbiology, Melaka Manipal Medical College, Manipal University, Manipal, Karnataka, India. Email: indira.bairy@manipal.edu

Received: 24 August 2016, Revised and Accepted: 29 August 2016

\section{ABSTRACT}

Objective: To study the clinico-microbiological profile of oral candidiasis in head and neck squamous cell cancer (HNSCC) patients undergoing curative radiotherapy (cRT).

Methods: Patients undergoing cRT and developing oral candidiasis were enrolled. Clinical features such as pain and xerostomia were recorded. Candida isolates from lesions were speciated using CHROMagar (Himedia Inc.), and antifungal susceptibility was determined using microbroth dilution (MBD). Patients were followed up to study the clinical course of infection.

Results: Of the 100 patients undergoing cRT, 79 developed oral candidiasis. Median duration to development of infection was 4 weeks (range: 1-6.5 weeks). Mucositis was observed in $76(96.2 \%)$ and xerostomia in 53 (67.1\%) patients; 61 patients (77.2\%) had symptoms attributable to candidiasis. However, there was no correlation between severity of infection and mucositis $(\mathrm{p}=0.84)$ or xerostomia $(\mathrm{p}=0.51)$. Candida albicans was the most frequent (47 patients, 59.4\%) isolate, followed by Candida tropicalis (23 patients; 29.1\%). All isolates were sensitive to nystatin, but fluconazole resistance/dose-dependent susceptibility was noted in 26 (32.9\%) isolates. Both Candida krusei and two of four Candida glabrata isolate exhibited fluconazole resistance. All patients received treatment for Candidiasis. On follow-up, 1 month after cRT, oral candidiasis resolved with gradual recovery of mucositis in all patients.

Conclusion: Candida albicans was the most common cause of oral Candidiasis in HNSCC cRT, and all isolates were susceptible to nystatin in-vitro. All lesions resolved with recovery from mucositis. In addition, as no patient developed systemic candidiasis, it appears that oral candidiasis though troublesome is curable with treatment.

Keywords: Radiation mucositis, CHROMagar, Microbroth dilution, Antifungal susceptibility.

(C) 2016 The Authors. Published by Innovare Academic Sciences Pvt Ltd. This is an open access article under the CC BY license (http://creativecommons org/licenses/by/4. 0/) DOI: http://dx.doi.org/10.22159/ajpcr.2016.v9s3.14870

\section{INTRODUCTION}

Candida species form a part of normal oral flora. Candida colonization rates of nearly $18 \%$ have been reported in healthy individuals [1]. However, various specific and non-specific defense mechanisms prevent the development of oral candidiasis. Colonization occurs whenever there is an imbalance in multiplication and clearance of Candida cells. This colonization can progress to infection whenever there is mucosal injury [1]. Mucositis and xerostomia are the most common complications of curative radiation therapy (cRT) for head and neck squamous cell carcinomas (HNSCC) and act in synergy to considerably increase the patient's risk of developing oropharyngeal candidiasis. The incidence rates of oral candidiasis in these patients have been reported to be as high as $77 \%[2]$.

While there are several studies that have reported on changes in candidial colonization during the course of cRT, few have studied the clinical course of overt infection and its response to treatment. In this study, we attempted to understand the microbiological profile of oropharyngeal candidiasis in patients undergoing cRT for HNSCC in our hospital and study their susceptibility patterns.

\section{METHODS}

This was a prospective study which included 100 patients who received cRT for HNSCC and developing oropharyngeal candidiasis. Ethical clearance was obtained from the Institutional Ethical Committee. The study group included both patients undergoing primary RT (with or without chemotherapy) and adjuvant RT following definitive surgery. Patients receiving palliative RT, suffering from oropharyngeal candidiasis prior to start of treatment, and/or those with Grade IV trismus were excluded from the study. Informed consent was obtained from all patients for participating in the study. None of the patients received prophylactic topical or systemic antifungal treatment. Patients were monitored on a weekly basis for the development of oral candidiasis. Personal and clinical details were obtained from all the patients with suspected candidiasis. The site, size, extent, and morphological patterns of the Candida lesions were recorded. The severity of acute mucositis and xerostomia secondary to RT was noted and graded according to Common Terminology Criteria for Adverse Events grading system (version 4.0) [3] for all patients. Oral swabs collected from the suspected lesions were subjected to Gram-stain and culture on Sabouraud dextrose agar. Plates were observed after $24 \mathrm{hrs}$ of incubation at $37^{\circ} \mathrm{C}$. Candida colonies grown were speciated using CHROMagar (HiMedia Inc.). The plates with no growth were further incubated at examined after $48 \mathrm{hrs}$. Conventional tests such as Dalmau plate technique and germ tube test were also performed for speciation. Mircobroth dilution (MBD) test was performed for determining antifungal susceptibility using Clinical and Laboratory Standards Institute (CLSI) M27-A2 reference standard [4]. Fluconazole and nystatin drug powders were obtained from Sigma-Aldrich.

Interpretation of MBD was done by appreciating the visible growth in each well. Growth was scored as per the scoring given in the reference standard guideline - Optically clear tube was graded as zero, slightly hazy growth as one, prominent decrease in turbidity as two, slight reduction in turbidity as three, and no reduction in turbidity as four. 
For fluconazole, lowest concentration at which score 2 was observed was considered as the minimum inhibitory concentration (MIC), whereas lowest concentration at which score 0 was observed was identified as MIC for nystatin. MIC value of $<8 \mu \mathrm{g} / \mathrm{ml}$ was considered as susceptible, $>32 \mu \mathrm{g} / \mathrm{ml}$ was considered resistant, and the isolates which had a MIC of $16-32 \mu \mathrm{g} / \mathrm{ml}$ were noted as susceptible dose dependent for fluconazole. There are no interpretive breakpoints available for nystatin by CLSI. In this study, the methodology described for amphotericin B by CLSI has been used to carry out MIC determination for nystatin. Patients were followed up for their response to therapy and until 1 month after completion of treatment to check any persistence of oral candidiasis.

\section{RESULTS}

Of the 100 patients who underwent definitive or adjuvant RT during the study period, 79 patients developed clinical evidence of candidiasis that was confirmed by culture. The mean age of the patients was 55 years (range: 29-86 years). General and clinical characteristics of the study population are described in Table 1 .

The time of onset of oral candidiasis during RT was variable, with a median duration for developing the infection being 4 weeks since the start of RT (range: 1-6.5 weeks). The average radiation dose received at the time of developing candidiasis was 41.2 Gy (range: 14 Gy-66 Gy) The clinical characteristics of Candida infection are summarized in Table 2. Majority (77\%) had symptoms secondary to mucositis, xerostomia, and/or candidiasis at the time of detection of infection. Pain on swallowing was the most common symptom (70\%), followed by burning sensation in the mouth $(34 \%)$, altered taste $(18 \%)$, and

Table 1: Demographic characteristics of the patients

\begin{tabular}{lll}
\hline Clinical parameter & Variable & N=100 (\%) \\
\hline Gender & Males & $79(79)$ \\
Site of primary & Females & $21(21)$ \\
& Oral cavity & $51(51)$ \\
& Oropharynx & $14(14)$ \\
& Hypopharynx & $20(20)$ \\
Tumor size & Others & $15(15)$ \\
& Tx & $6(6)$ \\
& T1 & $1(1)$ \\
& T2 & $30(30)$ \\
Tumor stage classification & T3 & $28(28)$ \\
& T4 & $35(35)$ \\
& I & $1(1)$ \\
& II & $17(17)$ \\
& III & $18(18)$ \\
& IVA & $55(55)$ \\
Grade of trismus & IVB & $9(9)$ \\
& $\leq$ I & $87(87)$ \\
& II & $7(7)$ \\
Concurrent chemotherapy & III & $53(6)$ \\
& Administered & $47(47)$ \\
\hline & Not administered & \\
& &
\end{tabular}

Table 2: Patient related variables at the onset of Candida infection $(\mathrm{N}=79)$

\begin{tabular}{llll}
\hline Clinical parameter & Variable & N (\%) & p value \\
\hline Severity of pain & 0 & $24(30.3)$ & 0.02 \\
& SII & $39(49.4)$ & \\
Grade of mucositis at & 0 & $16(20.3)$ & \\
onset of candidiasis & & $3(3.8)$ & 0.001 \\
& I-II & $56(70.9)$ & \\
Grade of xerostomia at & 0 & $20(25.3)$ & \\
onset of candidiasis & & $26(32.9)$ & 0.001 \\
& I & $47(59.5)$ & \\
& II & $6(7.6)$ & \\
\hline
\end{tabular}

nausea/vomiting (8\%). On analyzing with one sample Chi-square test, patients with candidiasis were significantly likely to experience at least some pain $(\mathrm{p}=0.02)$, mucositis $(\mathrm{p}=0.001)$, and xerostomia $(\mathrm{p}=0.001)$.

Growth was scanty in 36 (45.6\%), moderate in 24 (30.4\%), and heavy in $19(24 \%)$ samples. When the growth of Candida was evaluated in different grades of mucositis, it was seen that majority of positive cultures were obtained in Grade 1 and 2 mucositis. There was no statistical correlation between grades of mucositis and Candida growth $(\mathrm{p}=0.84)$. Even grades of trismus and xerostomia did not show any statistical correlation with degree of growth $(\mathrm{p}=0.79$ and 0.51 , respectively).

Speciation of the isolates revealed that Candida albicans were the most frequent (47 patients, 59.4\%), followed by Candida tropicalis (23 patients; 29.1\%). Other isolates included Candida glabrata (four patients), Candida krusei, and Candida parapsilosis (two patients each). One Candida famata was also identified.

All isolates were sensitive to nystatin by antifungal susceptibility testing. However, only 53 isolates were fluconazole susceptible. Fluconazole resistance was seen in 12 isolates and 14 exhibited dosedependent susceptibility (Table 3 ).

All patients who had microbiological evidence of candidiasis received treatment for oral candidiasis either in the form of topical or systemic antifungal therapy based on the clinical severity and grade of mucositis. Topical antifungal used was clotrimazole lozenges. Fluconazole was the preferred systemic agent and prescribed in 16 (89\%) of the 18 patients receiving any systemic therapy. Two patients received itraconazole. All patients showed clinical resolution of oral candidiasis following 1 month after completion of RT.

\section{DISCUSSION}

Oral candidiasis is a frequent complication of cRT for HNSCC, especially among oral cancers. This study was conducted to identify the burden and clinical features of oral candidiasis in these patients. The incidence of oral candidiasis during a typical 6-7 weeks course of cRT is quite high; $79 \%$ of our patients developed clinical evidence of oral candidiasis during the study period, which was confirmed by microbiological evidence in culture. The median time to onset of clinically evident candidiasis was 4 weeks in our study, and more than $70 \%$ of our patients had Grade II or higher oral mucositis at the time of development of infection. This reflects that the severity of mucositis increases with progress in RT which in turn increases the chances of development of oral candidiasis. Association of oral mucositis with the development of candidiasis is already established by some previous studies [5,6]. In one of such study of candidiasis among HNSCC patients, the incidence of oral thrush was much higher $(55.2 \%)$ in patients who received RT to oral region as compared to the group which did not receive RT to oral region (11\%) [5]. Similarly, in another study, candidiasis was associated with $\geq$ Grade II mucositis in $77 \%$ of the patients receiving RT for oral cancers [6]. However, we could not establish a significant relationship between grade of mucositis and severity of candidiasis. Similarly, there was no correlation between severity xerostomia and development of overt infection, even though xerostomia is a known risk factor for oral candidiasis. This is probably because most patients (67) had at least some degree of xerostomia at the time of developing infection. It has been reported that Candida colony forming unit counts were higher in patients with xerostomia when compared to patients with higher salivary flow rates [7]. In a patient with HNSCC receiving cRT, xerostomia and mucositis frequently coexist, making it difficult as to ascertain which toxicity is a greater predisposing factor. Coexistence of xerostomia and mucositis is highly likely to increase the risk of developing candidiasis; 55 of our patients with candidiasis had coexistent mucositis and xerostomia. It is also believed that overt candidiasis worsens the severity of acute radiation toxicity experienced by the patient, thus making the relationship between the toxicity of RT and oral candidiasis a vicious cycle. In a cohort study by Deng et al., 
Table 3: Fluconazole susceptibility among isolated species

\begin{tabular}{lllll}
\hline Candida isolate & Sensitive (\%) & Susceptible dose dependent (\%) & Resistant (\%) & Total \\
\hline Candida albicans & $33(70.2)$ & $9(19.1)$ & $5(10.7)$ & 47 \\
Candida tropicalis & $17(73.8)$ & $3(13.1)$ & $3(13.1)$ & 23 \\
Candida glabrata & $0(0)$ & $2(50)$ & $2(50)$ & 4 \\
Candida krusei & $0(0)$ & $0(0)$ & $2(100)$ & 2 \\
Candida parapsilosis & $2(100)$ & $0(0)$ & $0(0)$ & 1 \\
Candida famata & $1(100)$ & $0(0)$ & $0(0)$ & $12(15.2)$ \\
Total & $53(67.1)$ & $14(17.7)$ & & \\
\hline
\end{tabular}

Table 4: Studies reporting on oral Candida isolates among patients undergoing definitive RT

\begin{tabular}{|c|c|c|c|c|c|c|c|}
\hline Authors & Year & C. albicans $\%$ & C. tropicalis \% & C. parapsilosis $\%$ & C. glabrata $\%$ & C. krusie \% & Other species \% \\
\hline Belazi et al. [6] & 2004 & 51.1 & 3 & - & 8 & 3 & 1 \\
\hline Suryawanshi et al. [14] & 2012 & 84.62 & 2 & 1 & - & 2 & 1 \\
\hline de Freitas et al. [15] & 2013 & 16.2 & 27 & 16.2 & - & - & \\
\hline Bakki et al. [9] & 2014 & 26 & 4 & - & 1 & 4 & 2 \\
\hline Zhang et al. [11] & 2014 & 77 & 7 & 11 & 3.7 & - & - \\
\hline Yogitha et al. [9] & 2015 & 60.5 & 7 & 1 & 4 & 3 & \\
\hline Present study & 2016 & 59.4 & 29.1 & 2.5 & 5.3 & 2.5 & 1.2 \\
\hline
\end{tabular}

mouth soreness at the end of $4^{\text {th }}$ week of RT was significantly higher in patients with Candida infection [5].

Many different techniques have been used by researchers to sample oral Candida lesion such as oral swab, oral rinse, and salivary sampling $[8,9]$. We used sterile swabs to collect sample from lesions as we aimed at speciating the Candida isolates from suspected lesion for which oral rinse or salivary samples may not be ideal. However, the latter sampling methods are effective in estimating the colonization rates of Candida. Shrestha et al. have quantitatively estimated the colonization of Candida in patients undergoing head and neck radiation using oral rinse method [10]. They reported a colonization rate of $100 \%$ for C. albicans followed by C. glabrata and C. tropicalis. C. albicans is by far the most common species isolated in many studies involving patients with head and neck radiotherapy $[11,12]$. Even in the present study, C. albicans was the most common isolate (59.4\%). Among nonalbicans Candida (NAC), C. tropicalis was common (29.1) followed by C. parapsilosis, C. glabrata, and C. krusei. C. tropicalis is the common species among NAC group to cause bloodstream infections in patients with hematological malignancy [13]. The spectrum of Candida species isolated in patients undergoing head and neck radiotherapy by other studies is shown in Table 4.

Fluconazole resistance rates in Candida species are showing a steady increase. For instance, in a study on the antifungal susceptibility of C. tropicalis, fluconazole resistance was reported in $67 \%$ of isolates [16,17]. There are not many studies that have evaluated fluconazole resistance in Candida species isolated from HNSCC patients. Belazi et al. have reported a resistance rate of $34.7 \%$ in C. albicans, 66.6\% in C. glabrata, and $100 \%$ in C. krusei [6]. Our study has shown an overall resistance rate of $15.2 \%$ for fluconazole. The incidence of resistance was $10.7 \%$ in C. albicans, $13.7 \%$ in C. tropicalis, and $100 \%$ in $C$. krusei. This is expected as C. krusei is intrinsically resistant to fluconazole. Overall fluconazole resistance among NAC species was $8.8 \%$ in our study. In general, azole resistance is seen more commonly in NAC species. Moreover, antifungal resistance is more seen in Candida species isolated from systemic candidiasis or in isolates producing biofilm [18].

All isolates were susceptible for nystatin. There are no interpretive breakpoints available for nystatin by CLSI. In this study, the methodology described for amphotericin-B by CLSI has been used to carry out MIC determination for nystatin. Nystatin resistance is generally less in Candida species as seen in other studies. The resistance rates are usually zero; however, there are reported resistance of $1-6 \%$ in some of the studies [19-21].
A major limitation of the present study was the lack of systematic analysis of the treatment response to oral candidiasis as treatment received by the patients ranged from topical agents to systemic antifungals. Moreover, a study design involving follow-up cultures would have helped in understanding the microbiological recovery in addition to clinical recovery.

\section{CONCLUSION}

Oral candidiasis is of major concern in patients on cRT for HNSCC as it enhances the side effects of RT. However, it is rare for these patients to develop systemic disease secondary to the oral lesion. Treatment of oral thrush is important for improving oral hygiene and potentially ameliorating symptoms to decrease the morbidity. Speciation and knowing the antifungal susceptibility will help in the accurate treatment of candidiasis in these cases.

\section{REFERENCES}

1. Cannon RD, Chaffin WL. Oral colonization by Candida albicans. Crit Rev Oral Biol Med 1999;10(3):359-83.

2. Redding SW, Zellars RC, Kirkpatrick WR, McAtee RK, Caceres MA, Fothergill AW, et al. Epidemiology of oropharyngeal Candida colonization and infection in patients receiving radiation for head and neck cancer. J Clin Microbiol 1999;37(12):3896-900.

3. Department of Health and Human Services (US). Common Terminology Criteria for Adverse Events (CTCAE) Version 4.0 Washington, D.C. (US): National Institutes of Health, National Cancer Institute. Available from: http://evs.nci.nih.gov/ftp1/CTCAE/CTCAE 4.03 2010-06-14 QuickReference_5x7.pdf. [Last cited on 2016 Mar 28].

4. National Committee for Clinical Laboratory. Reference Method for Broth Dilution Antifungal Susceptibility Testing of Yeasts. Approved standard M27-A2. Wayne, PA: National Committee for Clinical Laboratory Standards; 2002.

5. Deng Z, Kiyuna A, Hasegawa M, Nakasone I, Hosokawa A, Suzuki M. Oral candidiasis in patients receiving radiation therapy for head and neck cancer. Otolaryngol Head Neck Surg 2010;143(2):242-7.

6. Belazi M, Velegraki A, Koussidou-Eremondi T, Andreadis D, Hini S, Arsenis $\mathrm{G}$, et al. Oral Candida isolates in patients undergoing radiotherapy for head and neck cancer: Prevalence, azole susceptibility profiles and response to antifungal treatment. Oral Microbiol Immunol 2004;19(6):347-51.

7. Torres SR, Peixoto CB, Caldas DM, Silva EB, Akiti T, Nucci M, et al. Relationship between salivary flow rates and Candida counts in subjects with xerostomia. Oral Surg Oral Med Oral Pathol Oral Radiol Endod 2002;93(2):149-54.

8. Yogitha PV, Lakshmi N, Lakshmi KR, Krishna PB, Cheemala SS Isolation and speciation of genus Candida in patients undergoing chemotherapy and radiotherapy for head and neck tumours. Int J Res Med Sci 2015;3(5):1189-94. 
9. Bakki SR, Kantheti LP, Kuruba KK, Poosarla C, Baddam VR, Mulakaluri RR. Candidal carriage, isolation and species variation in patients undergoing radiotherapy and chemotherapy for head and neck tumours. J NTR Univ Health Sci 2014;3(1):28-34.

10. Shrestha M, Boaz K, Srikant N, Shakya A. An assessment of candida colonization and species differentiation in head and neck cancer patients receiving radiation. J Nepal Health Res Counc 2014;12(28):156-61.

11. Zhang YY, Li AQ, Wang NN, Liu LN, Cui JL. Oral Candida species distribution in patients receiving radiotherapy for head and neck cancer. Shanghai Kou Qiang Yi Xue 2014;23(5):605-8.

12. Kurnatowski P, Moqbil S, Kaczmarczyk D. Signs, symptoms and the prevalence of fungi detected from the oral cavity and pharynx of radiotherapy subjects with head and neck tumors, and their susceptibility to chemotherapeutics. Ann Parasitol 2014;60(3):207-13.

13. Dangi YS, Soni ML, Namdeo KP. Oral candidiasis: A review. Int J Pharm Pharm Sci 2010;2(4):36-41.

14. Suryawanshi H, Ganvir SM, Hazarey VK, Wanjare VS. Oropharyngea candidosis relative frequency in radiotherapy patient for head and neck cancer. J Oral Maxillofac Pathol 2012;16(1):31-7.

15. de Freitas EM, Nobre SA, Pires MB, Faria RV, Batista AU, Bonan PR Oral Candida species in head and neck cancer patients treated by radiotherapy. Auris Nasus Larynx 2013;40(4):400-4.

16. Medeiros CI, Silva DD, Filho GG, Filho AA, Lima ED. Activity anti-C. tropicalis and effects of the combination of (s)-(-)-citronellal with four antifungal applied in vulvovaginal candidiasis. Int J Pharm Pharm Sci 2016;8(8):347-51.

17. Omar H, Garhy EL. An overview of the azoles of interest. Int J Curr Pharm Res 2014;7(1):1-6.

18. Deorukhkar SC, Saini S, Mathew S. Non-albicans Candida infection: An emerging Threat. Interdiscip Perspect Infect Dis 2014;2014:615958

19. Khan PA, Fatima N, Nabeela, Jahan S, Khan HM, Malik A. Antifunga susceptibility pattern of Candida isolates from a tertiary care hospita of North India: A five year study. Int J Curr Microbiol App Sci 2015;(Special issue 1):177-81.

20. Anna LM, Suárez LA, Zaragozí MT, Lancha MR, de Francisco NC, Andreu CM, et al. Susceptibility to nystatin of oral Candida isolates and its correlation with the response to treatment. Rev Cubana Med Trop 2010;62(3):237-44.

21. Mahmaudabadi AZ, Zarrin N, Fard MB. Antifungal susceptibility of Candida species isolated from candiduria. Jundishapur J Microbiol 2013;6(1):24-8 